\title{
MAGIC TeV gamma-ray observations of Markarian 421 during multiwavelength campaigns in 2006
}

J. Aleksić1 ${ }^{1}$ H. Anderhub ${ }^{2}$, L. A. Antonelli ${ }^{3}$, P. Antoranz ${ }^{4}$, M. Backes ${ }^{5}$, C. Baixeras ${ }^{6}$, S. Balestra ${ }^{4}$, J. A. Barrio ${ }^{4}$, D. Bastieri ${ }^{7}$, J. Becerra González ${ }^{8}$, J. K. Becker ${ }^{5}$, W. Bednarek ${ }^{9}$, A. Berdyugin ${ }^{10}$, K. Berger ${ }^{9}$, E. Bernardini ${ }^{11}$, A. Biland ${ }^{2}$, R. K. Bock ${ }^{12,7}$, G. Bonnoli ${ }^{13}$, P. Bordas ${ }^{14}$, D. Borla Tridon ${ }^{12}$, V. Bosch-Ramon ${ }^{14}$, D. Bose ${ }^{4}$, I. Braun ${ }^{2}$, T. Bretz ${ }^{15}$, D. Britzger ${ }^{12}$, M. Camara ${ }^{4}$, E. Carmona ${ }^{12}$, A. Carosi ${ }^{3}$, P. Colin ${ }^{12}$, S. Commichau ${ }^{2}$, J. L. Contreras ${ }^{4}$, J. Cortina ${ }^{1}$, M. T. Costado ${ }^{8,16}$, S. Covino ${ }^{3}$, F. Dazzi ${ }^{17,26}$, A. De Angelis ${ }^{17}$, E. de Cea del Pozo ${ }^{18}$, R. De los Reyes ${ }^{4,28}$, B. De Lotto ${ }^{17}$, M. De Maria ${ }^{17}$, F. De Sabata ${ }^{17}$, C. Delgado Mendez ${ }^{8,27}$, M. Doert ${ }^{5}$, A. Domínguez ${ }^{19}$,

D. Dominis Prester 20 , D. Dorner ${ }^{2}$, M. Doro ${ }^{7}$, D. Elsaesser ${ }^{15}$, M. Errando ${ }^{1}$, D. Ferenc ${ }^{21}$, M. V. Fonseca ${ }^{4}$, L. Font ${ }^{6}$, R. J. García López ${ }^{8,16}$, M. Garczarczyk ${ }^{8}$, M. Gaug ${ }^{8}$, N. Godinovic ${ }^{20}$, D. Hadasch ${ }^{18}$, A. Herrero ${ }^{8,16}$, D. Hildebrand ${ }^{2}$, D. Höhne-Mönch ${ }^{15}$, J. Hose ${ }^{12}$, D. Hrupec ${ }^{20}$, C. C. Hsu ${ }^{12}$, T. Jogler ${ }^{12}$, S. Klepser ${ }^{1}$, T. Krähenbühl2 ${ }^{2}$, D. Kranich ${ }^{2}$, A. La Barbera ${ }^{3}$, A. Laille ${ }^{21}$, E. Leonardo ${ }^{13}$, E. Lindfors ${ }^{10}$, S. Lombardi ${ }^{7}$, F. Longo ${ }^{17}$, M. López ${ }^{7}$, E. Lorenz ${ }^{2,12}$, P. Majumdar ${ }^{11}$, G. Maneva ${ }^{22}$, N. Mankuzhiyil ${ }^{17}$, K. Mannheim ${ }^{15}$, L. Maraschi $^{3}$, M. Mariotti $^{7}$, M. Martínez $^{1}$, D. Mazin ${ }^{1}$, M. Meucci ${ }^{13}$, J. M. Miranda ${ }^{4}$, R. Mirzoyan ${ }^{12}$, H. Miyamoto' ${ }^{12}$, J. Moldón ${ }^{14}$, M. Moles ${ }^{19}$, A. Moralejo ${ }^{1}$, D. Nieto ${ }^{4}$, K. Nilsson ${ }^{10}$, J. Ninkovic ${ }^{12}$, R. Orito ${ }^{12}$, I. Oya ${ }^{4}$, R. Paoletti ${ }^{13}$, J. M. Paredes ${ }^{14}$, S. Partini ${ }^{13}$, M. Pasanen ${ }^{10}$, D. Pascoli ${ }^{7}$, F. Pauss ${ }^{2}$, R. G. Pegna ${ }^{13}$, M. A. Perez-Torres ${ }^{19}$, M. Persic ${ }^{17,23}$, L. Peruzzo ${ }^{7}$, F. Prada ${ }^{19}$, E. Prandini ${ }^{7}$, N. Puchades ${ }^{1}$, I. Puljak ${ }^{20}$, I. Reichardt ${ }^{1}$, W. Rhode ${ }^{5}$, M. Ribó ${ }^{14}$, J. Rico ${ }^{24,1}$, M. Rissi ${ }^{2}$, S. Rügamer ${ }^{15}$, A. Saggion ${ }^{7}$, T. Y. Saito ${ }^{12}$, M. Salvati ${ }^{3}$, M. Sánchez-Conde ${ }^{19}$, K. Satalecka ${ }^{11}$, V. Scalzotto ${ }^{7}$, V. Scapin ${ }^{17}$, T. Schweizer ${ }^{12}$, M. Shayduk ${ }^{12}$, S. N. Shore ${ }^{25}$, A. Sierpowska-Bartosik ${ }^{9}$, A. Sillanpää ${ }^{10}$, J. Sitarek ${ }^{12,9}$, D. Sobczynska ${ }^{9}$, F. Spanier ${ }^{15}$, S. Spiro ${ }^{3}$, A. Stamerra ${ }^{13}$, B. Steinke ${ }^{12}$, N. Strah ${ }^{5}$, J. C. Struebig ${ }^{15}$, T. Suric ${ }^{20}$, L. Takalo ${ }^{10}$, F. Tavecchio ${ }^{3}$, P. Temnikov ${ }^{22}$, D. Tescaro ${ }^{1}$, M. Teshima ${ }^{12}$, D. F. Torres ${ }^{24,18}$, H. Vankov ${ }^{22}$, R. M. Wagner ${ }^{12}$, V. Zabalza ${ }^{14}$, F. Zandanel ${ }^{19}$, and R. Zanin ${ }^{1}$

\section{(Affiliations can be found after the references)}

Received 22 December 2009 / Accepted 19 May 2010

\section{ABSTRACT}

\begin{abstract}
Context. Wide-range spectral coverage of blazar-type active galactic nuclei is of paramount importance for understanding the particle acceleration mechanisms assumed to take place in their jets. The Major Atmospheric Gamma Imaging Cerenkov (MAGIC) telescope participated in three multiwavelength (MWL) campaigns, observing the blazar Markarian (Mkn) 421 during the nights of April 28 and 29, 2006 , and June 14, 2006. Aims. We analyzed the corresponding MAGIC very-high energy observations during 9 nights from April 22 to 30, 2006 and on June 14, 2006. We inferred light curves with sub-day resolution and night-by-night energy spectra.

Methods. MAGIC detects $\gamma$-rays by observing extended air showers in the atmosphere. The obtained air-shower images were analyzed using the standard MAGIC analysis chain.

Results. A strong $\gamma$-ray signal was detected from Mkn 421 on all observation nights. The flux $(E>250 \mathrm{GeV})$ varied on night-by-night basis between $(0.92 \pm 0.11) \times 10^{-10} \mathrm{~cm}^{-2} \mathrm{~s}^{-1}\left(0.57\right.$ Crab units) and $(3.21 \pm 0.15) \times 10^{-10} \mathrm{~cm}^{-2} \mathrm{~s}^{-1}$ (2.0 Crab units) in April 2006. There is a clear indication for intra-night variability with a doubling time of $36 \pm 10_{\text {stat }} \mathrm{min}$ on the night of April 29, 2006, establishing once more rapid flux variability for this object. For all individual nights $\gamma$-ray spectra could be inferred, with power-law indices ranging from 1.66 to 2.47 . We did not find statistically significant correlations between the spectral index and the flux state for individual nights. During the June 2006 campaign, a flux substantially lower than the one measured by the Whipple 10-m telescope four days later was found. Using a log-parabolic power law fit we deduced for some data sets the location of the spectral peak in the very-high energy regime. Our results confirm the indications of rising peak energy with increasing flux, as expected in leptonic acceleration models.
\end{abstract}

Key words. radiation mechanisms: non-thermal - BL Lacertae objects: individual: Mkn 421 - gamma rays: galaxies

\section{Introduction}

The active galactic nucleus (AGN) Markarian (Mkn) 421 was the first extragalactic source detected in the TeV energy range, using imaging atmospheric Cerenkov telescopes (IACTs, Punch et al. 1992; Petry et al. 1996). With a redshift of $z=0.030$ it is the closest known and, along with Mkn 501, the beststudied $\mathrm{TeV} \gamma$-ray emitting blazar ${ }^{1}$. So far, flux variations by

\footnotetext{
${ }^{1}$ See, e.g., http://www.mpp.mpg.de/ rwagner/sources/for an up-to-date list of VHE $\gamma$-ray sources.
}

more than one order of magnitude (e.g., Fossati et al. 2008), and occasional flux doubling times as short as 15 min (Gaidos et al. 1996; Aharonian et al. 2002; Schweizer et al. 2008) have been observed. Variations in the hardness of the $\mathrm{TeV} \gamma$-ray spectrum during flares were reported by several groups (e.g. Krennrich et al. 2002; Aharonian et al. 2005; Fossati et al. 2008). Simultaneous observations in the X-ray and very-high energy (VHE; $E \gtrsim 100 \mathrm{GeV}$ ) bands show strong evidence for correlated flux variability (Krawczynski et al. 2001; Błażejowski et al. 2005; Fossati et al. 2008). With a long history of observations, 
Mkn 421 is an ideal candidate for long-term and statistical studies of its emission (Tluczykont et al. 2007; Goebel et al. 2008a; Hsu et al. 2009).

Mkn 421 has been detected and studied at basically all wavelengths of the electromagnetic spectrum from radio waves up to VHE $\gamma$-rays. Its wide-range spectral energy distribution (SED) shows the typical double-peak structure of AGN. Mkn 421 is a so-called blazar. These constitute a rare subclass of AGNs with beamed emission closely aligned to our line of sight. In blazars, the low-energy peak at $\mathrm{keV}$ energies is thought to arise dominantly from synchrotron emission of electrons, while the origin of the high-energy ( $\mathrm{GeV}-\mathrm{TeV})$ bump is still debated. The SED is commonly interpreted as being due to the beamed, nonthermal emission of synchrotron and inverse-Compton radiation from ultrarelativistic electrons. These are assumed to be accelerated by shocks moving along the jets at relativistic bulk speed. For most of the observations, the SED can be reasonably well described by homogeneous one-zone synchrotron-self-Compton (SSC) models (e.g. Marscher \& Gear 1985; Maraschi et al. 1992; Costamante \& Ghisellini 2002). Hadronic models (Mannheim et al. 1996; Mücke et al. 2003), however, can also explain the observed features. A way to distinguish between the different emission models is to determine the positions, evolution and possible correlations (see, e.g., Wagner 2008b, for a review) of both peaks in the SED, using simultaneous, time-resolved data covering a broad energy range, e.g., as obtained in multiwavelength (MWL) observational campaigns.

In this Paper we present results from Major Atmospheric Gamma-ray Imaging Cerenkov (MAGIC) telescope VHE $\gamma$-ray observations of Mkn 421 during eight nights from April 22 to 30 , 2006, and on June 14, 2006. For most of the days, optical $R$-band observations were conducted with the KVA telescope. Simultaneous observations were performed by Suzaku (Mitsuda et al. 2007) and HESS, as well as by XMM-Newton (Jansen et al. 2001) on April 28 and 29, 2006, respectively. During both nights, we carried out particularly long, uninterrupted observations in the VHE energy band of $\approx 3 \mathrm{~h}$ duration each. An onset of activity in the X-ray band triggered an INTEGRAL-led target-of-opportunity (ToO) campaign, which took place from June 14-25, 2006 for a total of $829 \mathrm{ks}$ (Lichti et al. 2008). Within this campaign, MAGIC observed Mkn 421 at rather high zenith angles from 43 to 52 degrees in parallel with INTEGRAL on June 14, 2006.

In the following sections, we describe the data sets and the analysis applied to the VHE $\gamma$-ray data, the determination of spectra for all observation nights, and put the results into perspective with other VHE $\gamma$-ray observations of Mkn 421. The interpretation of these data in a MWL context is presented in Acciari et al. (2009) and subsequent papers.

VHE $\gamma$-ray observations in April and June 2006 have also been carried out by the Whipple telescope (Horan et al. 2009), by the VERITAS (Fegan 2008), and TACTIC (Yadav et al. 2007) collaborations, although not simultaneously with our observations.

\section{The MAGIC telescope}

The VHE $\gamma$-ray observations were conducted with the MAGIC telescope located on the Canary island La Palma (2200 $\mathrm{m}$ above sea level, $\left.28^{\circ} 45^{\prime} \mathrm{N}, 17^{\circ} 54^{\prime} \mathrm{W}\right)$. At the time of our observations in 2006, MAGIC was a single-dish 17-m $\varnothing$ instrument $^{2}$ for

\footnotetext{
2 Since 2009, MAGIC is a two-telescope stereoscopic system (Cortina et al. 2009).
}

the detection of atmospheric air showers induced by $\gamma$-rays. Its hexagonally-shaped camera with a field of view (FOV) of $\approx 3.5^{\circ}$ mean diameter comprises 576 high-sensitivity photomultiplier tubes (PMTs): 180 pixels of $0.2^{\circ} \varnothing$ surround the inner section of the camera of 394 pixels of $0.1^{\circ} \varnothing\left(=2.2^{\circ} \varnothing\right.$ FOV $)$. The trigger is formed by a coincidence of $\geq 4$ neighboring pixels. Presently the accessible trigger energy range (using the MAGIC standard trigger, Meucci et al. 2007) spans from 50-60 GeV (at small zenith angles) up to tens of TeV. Further details, telescope parameters, and performance information can be found in Baixeras et al. (2004); Cortina et al. (2005); Albert et al. (2008a).

\section{Observations and data analysis}

The observations were carried out during dark nights, employing the so-called wobble mode (Daum et al. 1997), in which two opposite sky directions, each $0.4^{\circ}$ off the source, are tracked alternatingly for $20 \mathrm{~min}$ each. The on-source data are defined by calculating image parameters with respect to the source position, whereas background control ("off") data are obtained from the same data set, but with image parameters calculated with respect to three positions, arranged symmetrically to the onsource region with respect to the camera center. The simultaneous measurement of signal and background makes additional background control data unnecessary. In order to avoid an unwanted contribution from source $\gamma$-events in the off sample, and to guarantee the statistical independence between the on and the off samples in the signal region, events included in the signal region of the on sample were excluded from the off sample and vice versa.

The data were analyzed following the standard MAGIC analysis procedure (Bretz \& Wagner 2003; Bretz \& Dorner 2008). After calibration (Albert et al. 2008c) and extracting the signal at the pulse maximum using a spline method, the air-shower images were cleaned of noise from night-sky background light by applying a three-stage image cleaning. The first stage requires a minimum number of 6 photoelectrons in the core pixels and 3 photoelectrons in the boundary pixels of the images (see, e.g. Fegan 1997). These tail cuts are scaled according to the larger size of the outer pixels of the MAGIC camera. Only pixels with at least two adjacent pixels with a signal arrival time difference lower than $1.75 \mathrm{~ns}$ survive the second cleaning stage. The third stage repeats the cleaning of the second stage, but requires only one adjacent pixel within the $1.75 \mathrm{~ns}$ time window.

The data were filtered by rejecting trivial background events, such as accidental noise triggers, triggers from nearby muons, or data taken during adverse atmospheric conditions (e.g., low atmospheric transmission). $12.7 \mathrm{~h}$ out of the total 15.0 hours' worth of data survived the latter quality selection and were used for further analysis.

We calculated image parameters (Hillas 1985) such as WIDTH, LENGTH, SIZE, CONC, M3LONG (the third moment of the light distribution along the major image axis), and LEAKAGE (the fraction of light contained in the outermost ring of camera pixels) for the surviving events. For the $\gamma /$ hadron separation, a SIZE-dependent parabolic cut in AREA $\equiv$ WIDTH $\times$ LENGTH $\times \pi$ was used (Riegel et al. 2005). The cut parameters for the assessment of the detection significance were optimized on Mkn 421 data from close-by days. For the data of June 14, 2006 at rather large zenith angles, data of Mkn 501 from October 2006 were used to determine the optimal cuts. Any significance in this work was calculated using Eq. (17) of Li \& Ma (1983) with $\alpha=1 / 3$. 
The primary $\gamma$-ray energies were reconstructed from the image parameters using a Random Forest regression method (Albert et al. 2008b, and references therein) trained with MonteCarlo simulated events (MCs, Knapp \& Heck 2004; Majumdar et al. 2005). The MC sample is characterized by a power-law spectrum between $10 \mathrm{GeV}$ and $30 \mathrm{TeV}$ with a differential spectral photon index of $\alpha=-2.6$, and a point-spread function resembling the experimental one. The events were selected to cover the same zenith distance range as the data. For the spectrum calculation, the area cut parameters were optimized to yield a constant MC cut efficiency of $90 \%$ over the whole energy range, increasing the $\gamma$-ray event statistics at the threshold.

The Mkn 421 observations presented here are among the first data taken by MAGIC after major hardware updates in April 2006 (Goebel et al. 2008b), which required us to thoroughly examine the data. Despite the hardware changes, the MAGIC subsystems performed as expected with the exception of an unstable trigger behavior for some PMTs, leading to a significant loss of events in one of the six sectors of the camera. In order to proceed with the data analysis with serenity and to estimate the effect caused by this inhomogeneity, a simple procedure was applied to the data: the expected number of events, as a function of energy, for the affected sector was estimated as the mean of the number of events in the other five sectors of the camera. (A homogeneous distribution of events through the six sectors is expected for normal conditions.) The difference between the expected and actually measured events was computed using the whole data sample in order to have sufficient statistics. We found a decrease of the differential photon flux of $5.7 \%$ between 250 and $400 \mathrm{GeV}, 4.6 \%$ between 400 and $650 \mathrm{GeV}, 2.2 \%$ between 650 and $1050 \mathrm{GeV}$ and $<1 \%$ for higher energies for the April 2006 data. Due to the higher zenith distance and energy threshold, the method was adapted for June 14, 2006 and yielded a decrease of $5.2 \%$ between 450 and $670 \mathrm{GeV}$ and $2.6 \%$ for higher energies. However the above mentioned effect is just an average one, with estimated flux errors of up to $6.6 \%$ showing up for individual nights.

To mitigate the effect of the inhomogeneity, instead of an (already increased) energy threshold of $250 \mathrm{GeV}$, higher thresholds of 350 or $450 \mathrm{GeV}$ were applied for some observation nights. In this way we made sure that the estimated systematic error remains within reasonable limits.

For the calculation of the individual light curves as well as for the overall April 2006 lightcurve, the flux between $250 \mathrm{GeV}$ and $350 \mathrm{GeV}$ was extrapolated for the nights with higher threshold. We assumed a power-law behavior in this energy range, with the spectral index determined for the first three energy bins of the whole April dataset (i.e., $\alpha=-2.08$ ). The flux normalization for each night has been determined at $500 \mathrm{GeV}$ by a fit to the first three differential spectral points, an energy range which is reliable for all affected nights.

Table 1 summarizes the analyzed data sets. The statistical significance of any detection is assessed by applying a cut in $\theta^{2}$, where $\theta$ is the angular distance between the expected source position and the reconstructed $\gamma$-ray arrival direction. The arrival directions of the showers in equatorial coordinates were calculated using the DISP method (Fomin et al. 1994; Lessard et al. 2001). We replaced the constant coefficient $\xi$ in the parameterization of DISP in the original approach by a term which is dependent on LEAKAGE, SIZE, and SLOPE,

$\xi=\xi_{0}+\xi_{1} \mathrm{SLOPE}+\xi_{2} \mathrm{LEAKAGE}+k \xi_{3}\left(\log _{10} \mathrm{SIZE}-\xi_{4}\right)^{2},(1)$

$k=0$ for $\log _{10}$ SIZE $<\xi_{4}$ and $k=1$ for $\log _{10}$ SIZE $\geq \xi_{4}$. The coefficients were determined using simulated data. The parameter
Table 1. Some characteristic parameters of the different data sets of the campaign.

\begin{tabular}{cccr}
\hline \hline Night & Observation Window [MJD] & $t_{\text {eff }}[\mathrm{h}]$ & ${\text { ZA }\left[{ }^{\circ}\right]}^{\circ}$ \\
\hline April 22, 2006 & $53847.97679-53848.01460$ & 0.76 & $18-28$ \\
April 24, 2006 & $53849.96428-53850.00669$ & 0.99 & $16-28$ \\
April 25, 2006 & $53850.92813-53850.99607$ & 1.53 & $10-26$ \\
April 26, 2006 & $53851.92862-53852.00383$ & 1.64 & $10-29$ \\
April 27, 2006 & $53852.93474-53853.00047$ & 1.42 & $12-28$ \\
April 28, 2006 & $53853.88173-53854.01394$ & 2.23 & $10-32$ \\
April 29, 2006 & $53854.89514-53855.04119$ & 2.78 & $9-41$ \\
April 30, 2006 & $53855.97283-53855.97906$ & 0.16 & $23-24$ \\
June 14, 2006 & $53900.91979-53900.95532$ & 0.80 & $43-52$ \\
\hline
\end{tabular}

Notes. $t_{\text {eff }}$ denotes the effective observation time. ZA gives the zenith angle range of the observations.

SLOPE is a measure for the longitudinal arrival time evolution of the shower in the camera plane similar to the time parameter GRADIENT in Aliu et al. (2009). Instead of defining the parameter from a fit to the arrival time distribution, however, SLOPE is determined as an analytical solution of the fit. Note that this new parametrization makes DISP and therefore $\theta^{2}$ source dependent.

All stated errors are statistical errors only; we estimate our systematic errors to be $16 \%$ for the energy scale, $11 \%$ for absolute fluxes and flux normalizations, and 0.2 for the spectral slopes (Albert et al. 2008a), not including the additional systematic flux errors mentioned above.

A second, independent analysis of the data yielded compatible results to those presented here.

\section{Results}

\subsection{Results for April 22-30, 2006}

MAGIC observed Mkn 421 from MJD 53847 to MJD 53855. During the observations, two MWL campaigns were carried out simultaneously with Suzaku and with XMM-Newton on MJD 53854 and MJD 53855, respectively. Mkn 421 was also observed as part of the monitoring program of the Whipple 10-m telescope (see Horan et al. 2009), albeit about $3.5 \mathrm{~h}$ after the MAGIC observations stopped, due to the different longitudes of the two instruments.

A strong $\gamma$-ray signal from the source was detected in all eight observation nights. In total, 3165 excess events were recorded over a background of 693 events for energies $>250 \mathrm{GeV}$, yielding an overall significance of $64.8 \sigma$. Mkn 421 exhibited an average flux of $F_{>250 \mathrm{GeV}}=(1.48 \pm 0.03) \times$ $10^{-10} \mathrm{~cm}^{-2} \mathrm{~s}^{-1}$. When compared to earlier observations (see, e.g. Albert et al. 2007a; Tluczykont et al. 2007; Goebel et al. 2008a; Steele et al. 2008), our observations indicate an elevated flux state of Mkn 421. We found high flux states in the nights of MJD $53850, F_{>250 \mathrm{GeV}}=(2.32 \pm 0.13) \times 10^{-10} \mathrm{~cm}^{-2} \mathrm{~s}^{-1}$, MJD 53853, $F_{>250 \mathrm{GeV}}=(3.21 \pm 0.15) \times 10^{-10} \mathrm{~cm}^{-2} \mathrm{~s}^{-1}$, and MJD 53 856, $F_{>250 \mathrm{GeV}}=(2.39 \pm 0.33) \times 10^{-10} \mathrm{~cm}^{-2} \mathrm{~s}^{-1}$ (Fig. 1) In the remaining nights (we assumed nights with fluxes below $1.6 \times 10^{-10} \mathrm{~cm}^{-2} \mathrm{~s}^{-1}$ as non-flare nights), Mkn 421 exhibited a low-flux average of $F_{>250 \mathrm{GeV}}=(1.09 \pm 0.03) \times 10^{-10} \mathrm{~cm}^{-2} \mathrm{~s}^{-1}$. The analysis results on a night-by-night basis are summarized in Table 2, and include the nightly numbers for excess and background events, significances, and average integral fluxes above $250 \mathrm{GeV}$ (where the nights with an energy cut of $350 \mathrm{GeV}$ where extrapolated down to $250 \mathrm{GeV}$, see Sect. 3 for details). 


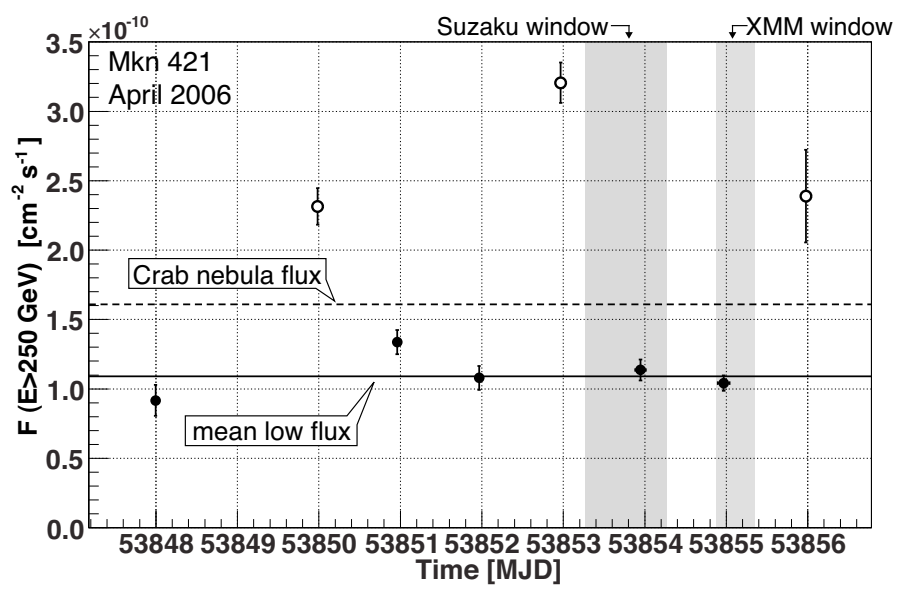

Fig. 1. VHE $(E>250 \mathrm{GeV})$ light curve for Mkn 421 observations in April 2006. The data points represent average nightly fluxes. The observation windows of the Suzaku (MJD 53 853.28-53 854.27) and XMM-Newton (MJD 53 854.87-53 855.35) MWL campaigns are marked by the gray-shaded areas. A "mean low flux" (solid line) was averaged over all data points below $1.6 \times 10^{-10} \mathrm{~cm}^{-2} \mathrm{~s}^{-1}$, i.e., excluding those data points marked by thin open circles. The dashed line gives the Crab nebula flux (Albert et al. 2008a) for comparison.

The results of a spectral fit based on a simple power law (PL) of the form

$\frac{\mathrm{d} F}{\mathrm{~d} E}=f_{0} \times 10^{-10} \mathrm{TeV}^{-1} \mathrm{~cm}^{-2} \mathrm{~s}^{-1}\left(\frac{E}{E_{0}}\right)^{-\alpha}$

are also shown.

The energy thresholds of the individual observations are also given in Table 2. As the analysis threshold is always lower than the applied energy cut, the latter one defines the energy threshold value.

The strong $\gamma$-ray signal allowed to infer light curves with a resolution below one hour for all of the observation nights, which are shown in Fig. 2 (see Table 4 for the light curve data). Most light curves are compatible with a constant flux during the nightly observation time (see Table 2 for all constant-fit $\chi_{\text {red }}^{2}$ values), while on MJD 53855 a clear intra-night variability is apparent. A fit with a constant function yields an unacceptable $\chi_{\text {red }}^{2}=41 / 7\left(P \approx 8 \times 10^{-5} \%\right)$ for this night, and the data suggest a flux halving time of $36 \pm 10_{\text {stat }}$ min. Note that this interesting observation window has also been covered by XMM-Newton observations in the X-ray band (Acciari et al. 2009).

\subsection{Results for June 14, 2006}

An onset of activity to $\approx 2$ times the average quiescent-flux level of Mkn 421 was measured in April 2006 by the RXTE allsky monitor (ASM) instrument. It triggered an INTEGRAL ToO campaign from June 14, 2006 to 25 for a total of $829 \mathrm{ks}$ (Lichti et al. 2008). This $>30 \mathrm{mCrab}$ flux remained until September 2006. During the 9-day campaign, Mkn 421 was targeted by various instruments in the radio, optical, X-ray and VHE wavebands. Results are reported in Lichti et al. (2008). On June 14, 2006, MAGIC observed Mkn 421 at rather high zenith angles in parallel with the OMC, JEM-X, and IBIS measurements aboard INTEGRAL. Further VHE coverage was provided by the Whipple 10-m telescope on June 18/19/21, 2006 (Lichti et al. 2008).

The MAGIC observations on June 14, 2006 lasted for $\approx 50 \mathrm{~min}$. The high zenith angles of 43 to 52 degrees of this observations and the previously mentioned inhomogeneities result in an energy threshold of $E_{\text {thresh }}=450 \mathrm{GeV}$. In spite of the overall rather difficult observational circumstances caused by the high zenith angle observations (Tonello 2006; Albert et al. 2006), a firm detection on the 7.5- $\sigma$ significance level was achieved.

The corresponding differential energy spectrum is shown in Fig. 3. Between $450 \mathrm{GeV}$ and $2.2 \mathrm{TeV}$, it can be described by a simple power-law of the form

$$
\frac{\mathrm{d} F}{\mathrm{~d} E}=(1.68 \pm 0.32) \times 10^{-11} \mathrm{TeV}^{-1} \mathrm{~cm}^{-2} \mathrm{~s}^{-1}\left(\frac{E}{1.0 \mathrm{TeV}}\right)^{-2.38 \pm 0.44}
$$

For comparison we also show the spectral points reported by the Whipple $10-\mathrm{m}$ telescope averaged over the nights of June 18/19/21, 2006. Generally, there might be systematic differences between the Whipple and MAGIC measurements. It could, however, be shown that such inter-instrument systematic effects are rather small and under control, e.g. those between MAGIC and HESS (Mazin et al. 2005). Particularly the Crab nebula spectra measured by Whipple and MAGIC agree quite well (Albert et al. 2008a). The Mkn 421 flux measured by the Whipple 10-m telescope four days after the MAGIC observation is substantially higher than our measurements (Fig. 3), pointing to a clear evolution of the source emission level within the INTEGRAL campaign.

\section{Discussion}

In leptonic acceleration models, e.g., SSC models, a shift of the high-energy peak (attributed to Inverse Compton radiation) in the spectral energy distribution towards higher energies with an increasing flux level is expected. In the VHE domain, such a shift can be traced by spectral hardening. Variations in the hardness of the TeV $\gamma$-ray spectrum during flares were reported by several groups (e.g., Krennrich et al. 2002; Aharonian et al. 2005; Fossati et al. 2008). We tested for a correlation of the spectral hardness with the flux level of the de-absorbed spectrum (i.e. after removing any attenuation effects caused by the Extragalactic Background Light [EBL], cf. Nikishov 1962; Gould \& Schréder 1966; Hauser \& Dwek 2001) in our data (Fig. 4), but found that the correlation neither can be described by a constant fit $\left(\chi_{\text {red }}^{2}=17 / 8, P \approx 3 \%\right.$ ) nor by a linear dependence of spectral hardness and flux level $\left(\chi_{\text {red }}^{2}=11 / 7, P \approx 12 \%\right.$ ), giving no clear preference for either. Although clear flux variations are present in the data set, the overall dynamical range of 3.9 in flux might be too small to see a significant spectral hardening with increasing flux.

The individual night-by-night spectra during the campaign in April 2006 are shown in Fig. 5. All spectral data points are summarized in Table 6. For the nights of April 22, 26, and 29, 2006 , there seems to be evidence for a resolved peak, but a likelihood ratio test (e.g., Mazin \& Goebel 2007) yields significant curvature only for April 27, 2006 ${ }^{3}$. We used a logarithmic curvature term, corresponding to a parabolic power-law $(\log -P)$ in a $\log \left(E^{2} \mathrm{~d} F / \mathrm{d} E\right)$ vs. $\log E$ representation (Massaro et al. 2004), and a power-law with exponential cutoff $(\mathrm{PL}+\mathrm{C})$ of the form

$$
\frac{\mathrm{d} F}{\mathrm{~d} E}=f_{0} \times 10^{-11} \mathrm{TeV}^{-1} \mathrm{~cm}^{-2} \mathrm{~s}^{-1}\left(\frac{E}{E_{0}}\right)^{-\left(\alpha+\beta \log _{10}\left(\frac{E}{E_{0}}\right)\right)}
$$

\footnotetext{
3 The respective log- $P$ probabilities are $83 \%, 48 \%, 73 \%$, and $96 \%$.
} 


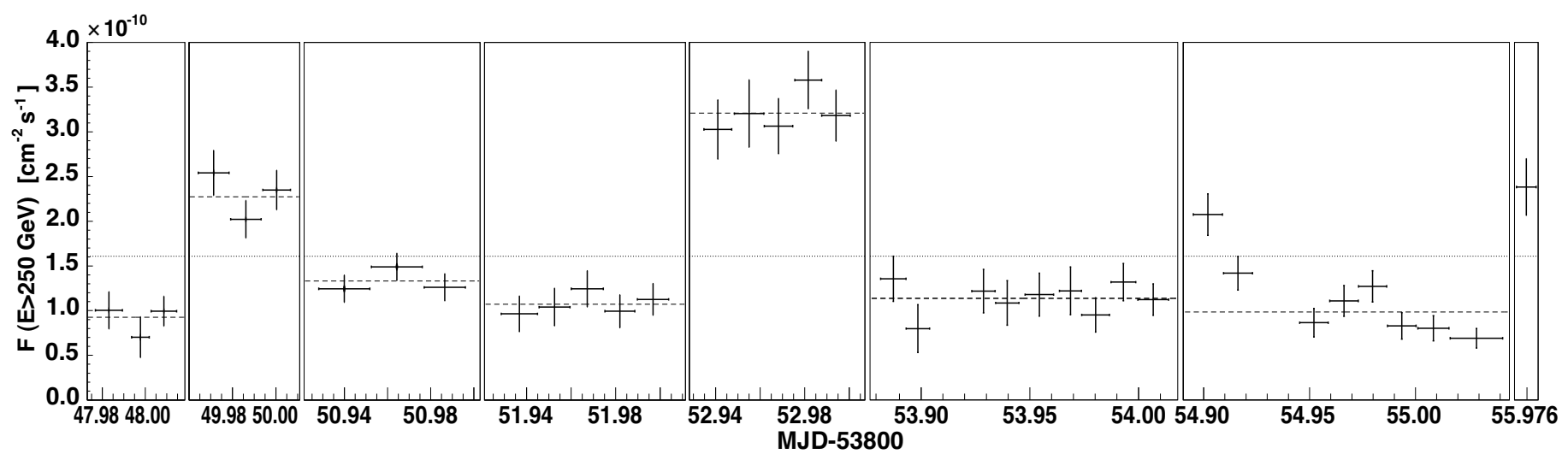

Fig. 2. VHE ( $E>250 \mathrm{GeV}$ ) light curve for Mkn 421 observations in April 2006. The dotted line represents the Crab nebula flux (Albert et al. 2008a), whereas the individual dashed lines show the result of a fit to the time bins (average nightly flux) of the corresponding nights.

Table 2. Analysis results.

\begin{tabular}{crrcccccrr}
\hline \hline Observation Night & $N_{\text {excess }}$ & $N_{\text {backgr. }}$ & \multicolumn{1}{c}{$S$} & $E_{\text {cut }}[\mathrm{GeV}]$ & $F\left(E>E_{\min }\right)$ & $\chi_{\text {red,const }}^{2}$ & $f_{0}$ & $\alpha$ & $\chi_{\text {red,PL }}^{2}$ \\
\hline April 22, 2006 & 100 & 29 & $10.9 \sigma$ & 350 & $0.92 \pm 0.11$ & $1.3 / 2$ & $0.98 \pm 0.15$ & $2.05 \pm 0.21$ & $2.1 / 2$ \\
April 24, 2006 & 419 & 69 & $25.0 \sigma$ & 250 & $2.32 \pm 0.13$ & $2.7 / 2$ & $2.45 \pm 0.14$ & $2.25 \pm 0.09$ & $2.0 / 3$ \\
April 25, 2006 & 342 & 83 & $20.8 \sigma$ & 250 & $1.34 \pm 0.09$ & $1.7 / 2$ & $1.43 \pm 0.09$ & $2.26 \pm 0.12$ & $0.24 / 3$ \\
April 26, 2006 & 225 & 62 & $16.4 \sigma$ & 350 & $1.08 \pm 0.09$ & $1.3 / 4$ & $1.21 \pm 0.11$ & $2.35 \pm 0.17$ & $0.41 / 2$ \\
April 27, 2006 & 615 & 56 & $33.5 \sigma$ & 350 & $3.21 \pm 0.15$ & $1.9 / 4$ & $3.37 \pm 0.18$ & $2.07 \pm 0.07$ & $4.8 / 4$ \\
April 28, 2006 & 311 & 75 & $19.9 \sigma$ & 350 & $1.14 \pm 0.08$ & $4.3 / 8$ & $1.32 \pm 0.10$ & $2.47 \pm 0.14$ & $0.65 / 2$ \\
April 29, 2006 & 514 & 169 & $23.7 \sigma$ & 250 & $1.04 \pm 0.06$ & $41 / 7$ & $1.14 \pm 0.06$ & $2.28 \pm 0.09$ & $2.0 / 4$ \\
April 30, 2006 & 69 & 11 & $10.3 \sigma$ & 250 & $2.39 \pm 0.33$ & - & $2.16 \pm 0.34$ & $1.66 \pm 0.20$ & $1.4 / 1$ \\
June 14, 2006 & 95 & 87 & $7.5 \sigma$ & 450 & $0.34 \pm 0.06$ & $2.4 / 1$ & $0.168 \pm 0.032$ & $2.38 \pm 0.44$ & $1.5 / 2$ \\
\hline
\end{tabular}

Notes. Number of excess $\left(N_{\text {excess }}\right)$ and background $\left(N_{\text {backgr. }}\right)$ events, resulting significances $S$, lower cuts in event energy, integral fluxes $F$ above $E_{\min }=250 \mathrm{GeV}$ for the April 2006 data and $E_{\min }=450 \mathrm{GeV}$ for the June 14,2006 data (in units of $10^{-10} \mathrm{~cm}^{-2} \mathrm{~s}^{-1}$ ), fit quality of a constant-flux fit to the individual observation nights (see Fig. 2), and power-law fit results for the differential energy spectra of $\mathrm{d} F / \mathrm{d} E=f_{0} \cdot\left(E / E_{0}\right)^{-\alpha}$ with $E_{0}=0.5 \mathrm{TeV}$ for the April 2006 data and $E_{0}=1.0 \mathrm{TeV}$ for the June 14, 2006 data, respectively; $f_{0}$ in units of $10^{-10} \mathrm{TeV}^{-1} \mathrm{~cm}^{-2} \mathrm{~s}^{-1}$.

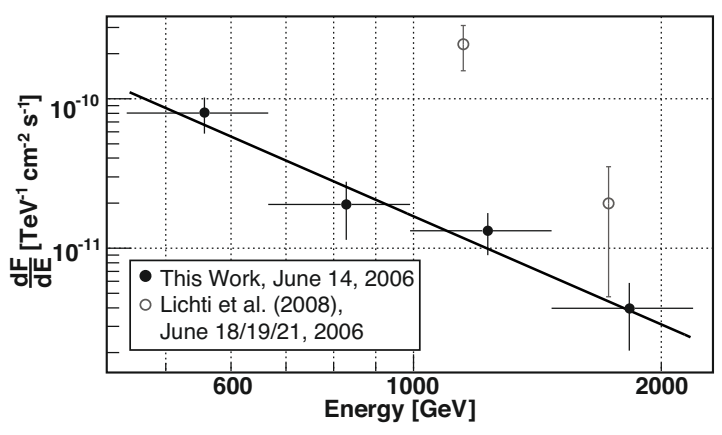

Fig. 3. Differential photon spectrum for Mkn 421 for the observation night of June 14, 2006 (black data points). A power-law fit to the spectrum results in a spectral slope of $\alpha=-2.38 \pm 0.44$ (see Table 2 for the fit results). Also shown are spectral points measured with the Whipple 10-m telescope (Lichti et al. 2008) during June 18-21, 2006.

and

$\frac{\mathrm{d} F}{\mathrm{~d} E}=f_{0} \times 10^{-11} \mathrm{TeV}^{-1} \mathrm{~cm}^{-2} \mathrm{~s}^{-1}\left(\frac{E}{E_{0}}\right)^{-\alpha} \exp \left(\frac{-E}{E_{\text {cut }}}\right)$,

respectively. The likelihood ratio test results in a clear preference towards a $\log -P$ or a $\mathrm{PL}+\mathrm{C}$ compared to a simple power-law with a probability of $\approx 96 \%$ for both of them. The $\chi_{\text {red }}^{2}$ values for PL, $\log -P$, and $\mathrm{PL}+\mathrm{C}$ fits on the individual night-by-night spectra in Fig. 5 are given in Table 5. Also the high statistics data sets defined by combining all data from April, all data from the five low-state nights and all data from the three high-state nights,

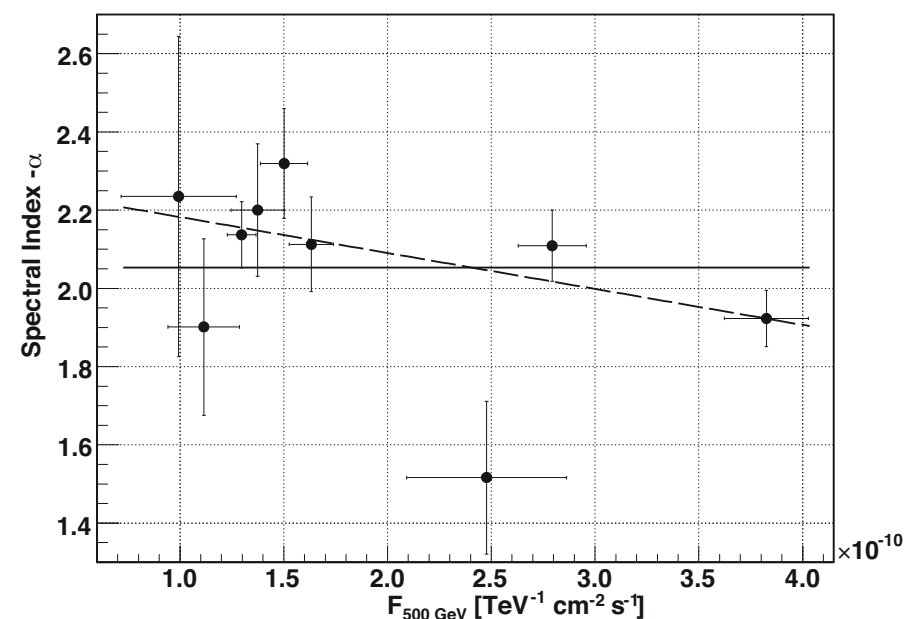

Fig. 4. Spectral index vs. flux at $0.5 \mathrm{TeV}$ deduced from a simple powerlaw fit after EBL de-absorption for Mkn 421. The $\chi_{\text {red }}^{2}$ for a constant fit (spectral index uncorrelated with flux level; solid line) amounts to $17 / 8$ $(P \approx 3 \%)$, while a linear correlation (dashed line) has a $\chi_{\text {red }}^{2}=11 / 7$, equal to $P \approx 12 \%$.

clearly showed evidence for a parabolic or cutoff shape of the spectra. The results of the fits and the probability of a likelihood ratio test are given in Table 3 . For all these nights our data did not allow to prefer one model over the other. The fact that all of the high statistics data sets show a curved spectral shape is an 

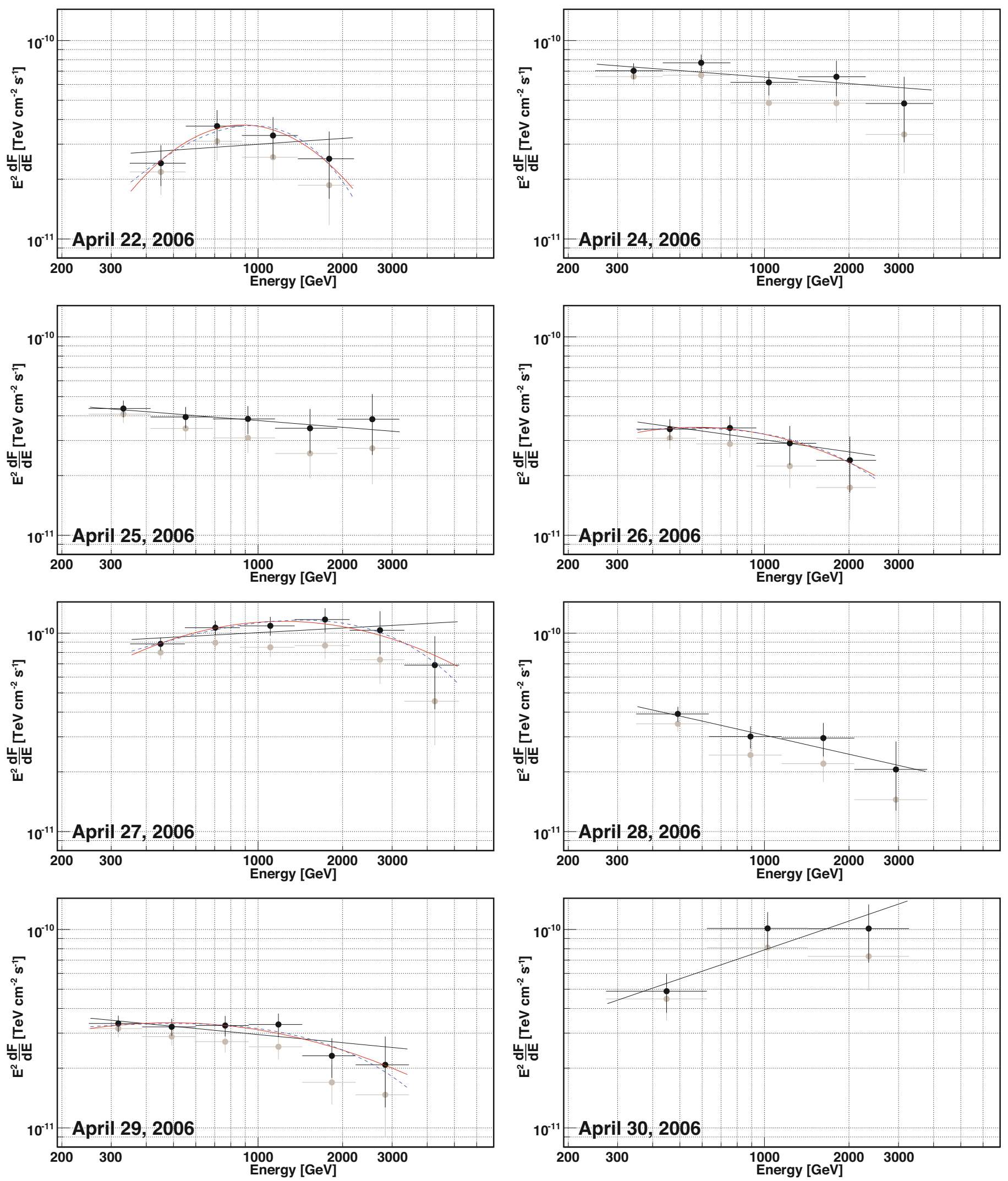

Fig. 5. Differential energy spectra for Mkn 421 for April 2006 before (gray points) and after (black points) correcting for EBL absorption. For the apparently hard spectra on April 22, 26, 27, and 29, 2006, log-P (Eq. (4)) and PL+C (Eq. (5)) fits were performed (red solid and blue dashed curves, respectively).

indication of this feature being always visible for Mkn 421 and hence source intrinsic.

Page 6 of 10
The curved power laws enable to locate a peak in the deabsorbed spectrum at $E_{\text {peak }}=E_{0} 10^{(2-\alpha) /(2 \beta)}$ for the log- $P$ and at $E_{\text {peak }}=(2-\alpha) E_{\text {cut }}$ if $\alpha<2$ for the PL+C fit. For simplicity 
Table 3. Special fit results.

\begin{tabular}{|c|c|c|c|c|c|c|c|c|}
\hline Data Set & Used Fit & $f_{0}$ & $\alpha^{\left({ }^{\prime}\right)}$ & $\beta$ & $E_{\text {cut }}[\mathrm{TeV}]$ & $\chi_{\text {red,fit }}^{2}$ & Likelihood & $E_{\text {peak }}[\mathrm{TeV}]$ \\
\hline \multirow{4}{*}{ April 27, 2006} & $\mathrm{PL}$ & $9.54 \pm 0.52$ & $1.92 \pm 0.07$ & \multirow{4}{*}{$0.59 \pm 0.29$} & & $5.3 / 4$ & & \\
\hline & $\log -P$ & $9.35 \pm 0.55$ & $1.54 \pm 0.19$ & & & $0.48 / 3$ & $96 \%$ & $1.2 \pm 0.7$ \\
\hline & $\log -P$ apex & $11.5 \pm 0.9$ & $0.26 \pm 0.17$ & & & $0.48 / 3$ & $96 \%$ & $1.2 \pm 0.2$ \\
\hline & $\mathrm{PL}+\mathrm{C}$ & $11.3 \pm 1.2$ & $1.44 \pm 0.24$ & & $2.6 \pm 1.3$ & $0.34 / 3$ & $96 \%$ & $1.4 \pm 1.0$ \\
\hline \multirow{4}{*}{ All April Data } & PL & $4.53 \pm 0.07$ & $2.07 \pm 0.04$ & \multirow{4}{*}{$0.39 \pm 0.11$} & & $16 / 5$ & & \\
\hline & $\log -P$ & $4.75 \pm 0.12$ & $1.89 \pm 0.06$ & & & $1.2 / 4$ & $99 \%$ & $0.69 \pm 0.14$ \\
\hline & $\log -P$ apex & $4.84 \pm 0.16$ & $0.41 \pm 0.11$ & & & $1.2 / 4$ & $99 \%$ & $0.69 \pm 0.06$ \\
\hline & $\mathrm{PL}+\mathrm{C}$ & $5.36 \pm 0.31$ & $1.77 \pm 0.09$ & & $3.6 \pm 1.1$ & $1.8 / 4$ & $99 \%$ & $0.80 \pm 0.42$ \\
\hline \multirow{4}{*}{ High-State Nights } & $\mathrm{PL}$ & $8.19 \pm 0.28$ & $1.93 \pm 0.05$ & \multirow{4}{*}{$0.29 \pm 0.15$} & & $6.0 / 4$ & & \\
\hline & $\log -P$ & $8.46 \pm 0.32$ & $1.79 \pm 0.09$ & & & $2.0 / 3$ & $94 \%$ & $1.1 \pm 0.6$ \\
\hline & $\log -P$ apex & $9.21 \pm 0.47$ & $0.52 \pm 0.17$ & & & $2.0 / 3$ & $94 \%$ & $1.1 \pm 0.3$ \\
\hline & $\mathrm{PL}+\mathrm{C}$ & $9.02 \pm 0.64$ & $1.75 \pm 0.12$ & & $6.1 \pm 4.0$ & $3.1 / 3$ & $91 \%$ & $1.5 \pm 1.2$ \\
\hline \multirow{4}{*}{ Low-State Nights } & PL & $3.39 \pm 0.10$ & $2.17 \pm 0.05$ & \multirow{4}{*}{$0.38 \pm 0.17$} & & $6.6 / 4$ & & \\
\hline & $\log -P$ & $3.55 \pm 0.13$ & $2.02 \pm 0.08$ & & & $1.1 / 3$ & $97 \%$ & $0.48 \pm 0.12$ \\
\hline & $\log -P$ apex & $3.55 \pm 0.13$ & $0.41 \pm 0.16$ & & & $1.1 / 3$ & $97 \%$ & $0.48 \pm 0.12$ \\
\hline & $\mathrm{PL}+\mathrm{C}$ & $4.15 \pm 0.40$ & $1.85 \pm 0.15$ & & $2.9 \pm 1.3$ & $0.75 / 3$ & $97 \%$ & $0.45 \pm 0.47$ \\
\hline
\end{tabular}

Notes. Results of a power-law fit (Eq. (2)), a log-parabolic fit (Eq. $\left.(4) \times\left(E / E_{0}\right)^{2}\right)$, a log-parabolic fit in apex form (Eq. (6)) and a power-law fit with an exponential cutoff $\left(\left(\right.\right.$ Eq. $\left.(5) \times\left(E / E_{0}\right)^{2}\right)$ in $E^{2} \mathrm{~d} F / \mathrm{d} E$ after EBL de-absorption for special data sets. $f_{0}$ is given in units of $10^{-11} \mathrm{TeV}^{-1} \mathrm{~cm}^{-2} \mathrm{~s}^{-1}$; $\alpha, \alpha^{\prime}, \beta, E_{\text {cut }}$ and $E_{\text {peak }}$ are the fit parameters as stated in the text, and Likelihood denotes the probability of a likelihood ratio test. The on/off normalization factor is $1 / 3, E_{0}=0.5 \mathrm{TeV}$.

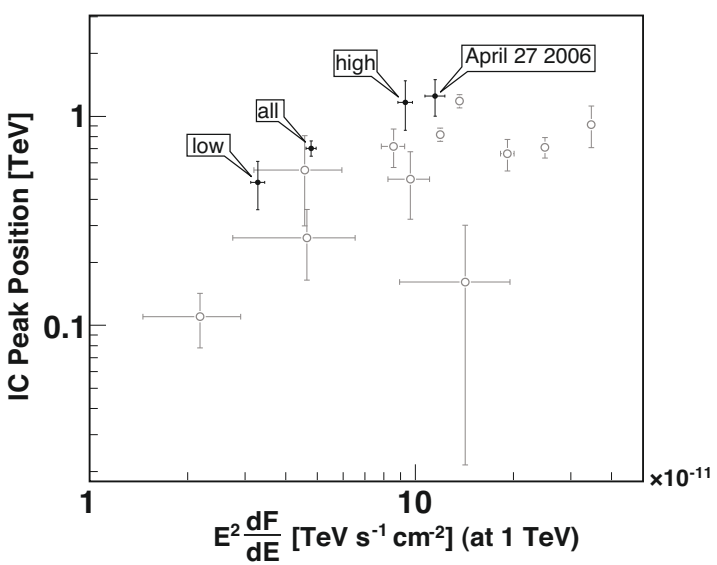

Fig. 6. Derived peak position using the $\log -P$ (Eq. (6)) versus flux at $1 \mathrm{TeV}$ for the data sets presented in Table 3. Historical data, taken from Albert et al. (2007a), are shown in gray. Our data confirm the indication of a correlation between the two parameters.

we determined $E_{\text {peak }}$ of the $\log -P$ by using the apex form of the parabola in a logarithmic representation:

$\log _{10} \frac{\mathrm{d} F}{\mathrm{~d} E}=\log _{10} f_{0}+\log _{10} \alpha^{\prime}\left(\log _{10}\left(\frac{E}{E_{0}} / \frac{E_{\text {peak }}}{E_{0}}\right)\right)^{2}$

which naturally yields both $E_{\text {peak }}$ and the flux at the peak, $f_{0}$, respectively. Additionally, the spectral cutoff is naturally obtained from the PL+C fit as the fit parameter $E_{\text {cut }}$. The results are shown in Table 3 . The values of $E_{\text {peak }}$ as determined using the log- $P$ and the $\mathrm{PL}+\mathrm{C}$ were compatible with each other for the data sets averaging several nights and showed indications for an increase of the peak energy with rising flux level, as predicted if the VHE radiation were due to SSC mechanisms. We compare our results with historical values taken from Albert et al. (2007a) in Fig. 6. Our data confirm the previously suggested correlation.

The observation of a relation between flux (and thus, fluence) and the position of the VHE peak in the SED could be signalling a relation similar to the one suggested by Amati et al. (2002) and observed by Sakamoto et al. (2006) for gamma-ray bursts. Since the $\mathrm{TeV} \gamma$-ray production is assumed to take place in a relativistic jet, and many of the same radiative processes are involved (on a larger scale, of course) it might be a similar (or related) mechanism at work on a different scale. A trend towards a relation between flux and spectral index in the TeV energy range has also been noted by Wagner (2008a), studying 17 known TeV blazars, and by Tramacere (2010) in the X-ray band, after a deep spectral analysis of all Swift observations of Mrk 421 between April and July 2006.

Although the peak energy measured on April 27, 2006 exceeds that of the All April Data and Low-State data set, it is, despite having a higher flux, comparable with that derived for the High-State data set. This discrepancy in terms of the expected behaviour in SSC models can be explained with the different nature of the data sets: the April 27, 2006 data represent a rather particular, $1.4 \mathrm{~h}$ long episode of an individual flare event, whereas the High-State data set is an average of three individual flares. Due to the sparse sampling, most probably each of these observations caught different epochs of the individual flare evolutions, during which the spectral shape can change considerably in terms of spectral index and curvature (see, e.g., Katarzyński et al. 2006). Hence the two data sets are not necessarily directly comparable.

The values of the derived cutoff energies are also suggesting this behavior, showing, with the exception of April 27, 2006, an increase with rising flux, thus indicating a source-intrinsic rather than a cosmological reason for the cutoff feature. This is in accordance with the Kneiske \& Dole (2008) lower-limit model, predicting an EBL cutoff for Mkn 421 at around $13 \mathrm{TeV}$.

In Fig. 7, we compare "historical" spectra measured between 1998 and 2005 with the low-state and high-state spectra derived from the observations reported here. It is obvious that our low-state spectrum represents one of the lowest flux states ever measured in VHE for Mkn 421, whereas the high state spectrum shows no exceptionally high flux level of this source. Both spectra are harder than historical spectra with comparable flux levels, in particular harder than the VERITAS spectrum (Donnarumma et al. 2009), enabling one of the best measurements of the turnover of the SED in a low flux state. While a 
Table 4. Light curve data.

\begin{tabular}{|c|c|}
\hline $\begin{array}{c}\text { Observation } \\
\text { [MJD] }\end{array}$ & $\begin{array}{r}F_{>250 \mathrm{GeV}} \\
{\left[10^{-10} \mathrm{~cm}^{-2} \mathrm{~s}^{-1}\right]}\end{array}$ \\
\hline \multicolumn{2}{|c|}{$2006 / 04 / 22$} \\
\hline 53847.98307 & $1.00 \pm 0.21$ \\
\hline 53847.99775 & $0.70 \pm 0.23$ \\
\hline 53848.00867 & $0.99 \pm 0.17$ \\
\hline \multicolumn{2}{|c|}{$2006 / 04 / 24$} \\
\hline 53849.97136 & $2.56 \pm 0.25$ \\
\hline 53849.98618 & $2.04 \pm 0.21$ \\
\hline 53850.00033 & $2.37 \pm 0.22$ \\
\hline \multicolumn{2}{|c|}{$2006 / 04 / 25$} \\
\hline 53850.93996 & $1.24 \pm 0.15$ \\
\hline 53850.96431 & $1.49 \pm 0.15$ \\
\hline 53850.98652 & $1.26 \pm 0.15$ \\
\hline \multicolumn{2}{|c|}{$2006 / 04 / 26$} \\
\hline 53851.93677 & $0.97 \pm 0.20$ \\
\hline 53851.95255 & $1.04 \pm 0.21$ \\
\hline 53851.96726 & $1.25 \pm 0.20$ \\
\hline 53851.98190 & $1.00 \pm 0.18$ \\
\hline 53851.99680 & $1.13 \pm 0.18$ \\
\hline \multicolumn{2}{|c|}{$2006 / 04 / 27$} \\
\hline 53852.94098 & $3.01 \pm 0.33$ \\
\hline 53852.95502 & $3.19 \pm 0.38$ \\
\hline 53852.96823 & $3.05 \pm 0.31$ \\
\hline 53852.98159 & $3.57 \pm 0.32$ \\
\hline 53852.99406 & $3.17 \pm 0.28$ \\
\hline \multicolumn{2}{|c|}{$2006 / 04 / 28$} \\
\hline 53853.88754 & $1.36 \pm 0.25$ \\
\hline 53853.89880 & $0.80 \pm 0.27$ \\
\hline 53853.92893 & $1.22 \pm 0.24$ \\
\hline 53853.93984 & $1.09 \pm 0.25$ \\
\hline 53853.95457 & $1.18 \pm 0.24$ \\
\hline 53853.96887 & $1.22 \pm 0.27$ \\
\hline 53853.98040 & $0.95 \pm 0.19$ \\
\hline 53853.99316 & $1.32 \pm 0.21$ \\
\hline 53854.00687 & $1.12 \pm 0.18$ \\
\hline \multicolumn{2}{|c|}{$2006 / 04 / 29$} \\
\hline 53854.90199 & $2.07 \pm 0.23$ \\
\hline 53854.91620 & $1.42 \pm 0.19$ \\
\hline 53854.95206 & $0.86 \pm 0.16$ \\
\hline 53854.96625 & $1.11 \pm 0.17$ \\
\hline 53854.97974 & $1.27 \pm 0.18$ \\
\hline 53854.99354 & $0.83 \pm 0.15$ \\
\hline 53855.00847 & $0.80 \pm 0.14$ \\
\hline 53855.02879 & $0.69 \pm 0.11$ \\
\hline \multicolumn{2}{|c|}{$2006 / 04 / 30$} \\
\hline 53855.97595 & $2.39 \pm 0.33$ \\
\hline \multicolumn{2}{|c|}{$2006 / 06 / 14$} \\
\hline 53900.92797 & $0.45 \pm 0.09$ \\
\hline 53900.94585 & $0.26 \pm 0.08$ \\
\hline
\end{tabular}

previous observation yielded a rather flat spectrum in the VHE regime (Aharonian et al. 2002), we conclude that we measured a rather clear peak (flat structure in the SED). The low-state spectrum has a shape similar to the one measured by HEGRA CT1, although at an approximately three times lower flux level. The high-state spectral shape resembles the high-state Whipple spectrum, which in turn has an about three times higher flux. This tendency can also be seen in Fig. 6, which shows that the fluxes we derive are systematically lower than historical measurements for comparable peak energies. Within the SSC framework this difference in flux for comparable spectral shapes can be caused by, e.g., a lower number of electrons with the same energy distribution as in the high-flux case.
Table 5. $\chi_{\text {red }}^{2}$ values for the PL, log-P, and PL $+C$ fits performed in Fig. 5.

\begin{tabular}{lcccccccc}
\hline \hline & 22 & 24 & 25 & 26 & 27 & 28 & 29 & 30 \\
\hline PL & $2.2 / 2$ & $1.9 / 3$ & $0.21 / 3$ & $0.47 / 2$ & $5.3 / 4$ & $0.59 / 2$ & $2.3 / 4$ & $1.5 / 1$ \\
$\log -P$ & $0.14 / 1$ & & & $0.041 / 1$ & $0.48 / 3$ & & $1.1 / 3$ & \\
PL+C & $0.27 / 1$ & & & $0.076 / 1$ & $0.34 / 3$ & & $0.87 / 3$ & \\
\hline
\end{tabular}

Notes. The columns represent days in April 2006.

Table 6. Energy spectra for all observation nights under study after EBL de-absorption.

\begin{tabular}{|c|c|c|}
\hline \multicolumn{2}{|c|}{$\begin{array}{c}E \text { bounds } \\
{[\mathrm{GeV}]}\end{array}$} & $\begin{array}{c}\text { Flux } \\
{\left[\mathrm{TeV} \mathrm{cm}^{-2} \mathrm{~s}^{-1}\right]}\end{array}$ \\
\hline \multicolumn{3}{|c|}{$2006 / 04 / 22$} \\
\hline 350 & 554 & $(2.39 \pm 0.56) \times 10^{-11}$ \\
\hline 554 & 877 & $(3.67 \pm 0.75) \times 10^{-11}$ \\
\hline 877 & 1389 & $(3.29 \pm 0.79) \times 10^{-11}$ \\
\hline 1389 & 2200 & $(2.51 \pm 0.93) \times 10^{-11}$ \\
\hline \multicolumn{3}{|c|}{$2006 / 04 / 24$} \\
\hline 250 & 435 & $(7.00 \pm 0.64) \times 10^{-11}$ \\
\hline 435 & 758 & $(7.69 \pm 0.76) \times 10^{-11}$ \\
\hline 758 & 1320 & $(6.12 \pm 0.86) \times 10^{-11}$ \\
\hline 1320 & 2297 & $(6.54 \pm 1.33) \times 10^{-11}$ \\
\hline 2297 & 4000 & $(4.80 \pm 1.74) \times 10^{-11}$ \\
\hline \multicolumn{3}{|c|}{$2006 / 04 / 25$} \\
\hline 250 & 416 & $(4.36 \pm 0.43) \times 10^{-11}$ \\
\hline 416 & 693 & $(3.95 \pm 0.48) \times 10^{-11}$ \\
\hline 693 & 1154 & $(3.86 \pm 0.62) \times 10^{-11}$ \\
\hline 1154 & 1922 & $(3.47 \pm 0.87) \times 10^{-11}$ \\
\hline 1922 & 3200 & $(3.85 \pm 1.31) \times 10^{-11}$ \\
\hline \multicolumn{3}{|c|}{$2006 / 04 / 26$} \\
\hline 350 & 572 & $(3.41 \pm 0.41) \times 10^{-11}$ \\
\hline 572 & 935 & $(3.46 \pm 0.49) \times 10^{-11}$ \\
\hline 935 & 1529 & $(2.90 \pm 0.65) \times 10^{-11}$ \\
\hline 1529 & 2500 & $(2.38 \pm 0.75) \times 10^{-11}$ \\
\hline \multicolumn{3}{|c|}{$2006 / 04 / 27$} \\
\hline 350 & 549 & $(8.83 \pm 0.66) \times 10^{-11}$ \\
\hline 549 & 860 & $(1.07 \pm 0.09) \times 10^{-10}$ \\
\hline 860 & 1349 & $(1.09 \pm 0.12) \times 10^{-10}$ \\
\hline 1349 & 2115 & $(1.17 \pm 0.17) \times 10^{-10}$ \\
\hline 2115 & 3317 & $(1.03 \pm 0.26) \times 10^{-10}$ \\
\hline 3317 & 5200 & $(6.89 \pm 2.76) \times 10^{-11}$ \\
\hline \multicolumn{3}{|c|}{$2006 / 04 / 28$} \\
\hline 350 & 635 & $(3.92 \pm 0.34) \times 10^{-11}$ \\
\hline 635 & 1153 & $(3.01 \pm 0.39) \times 10^{-11}$ \\
\hline 1153 & 2093 & $(2.96 \pm 0.57) \times 10^{-11}$ \\
\hline 2093 & 3800 & $(2.06 \pm 0.79) \times 10^{-11}$ \\
\hline \multicolumn{3}{|c|}{$2006 / 04 / 29$} \\
\hline 250 & 387 & $(3.37 \pm 0.32) \times 10^{-11}$ \\
\hline 387 & 600 & $(3.24+0.32) \times 10^{-11}$ \\
\hline 600 & 929 & $(3.29 \pm 0.39) \times 10^{-11}$ \\
\hline 929 & 1438 & $(3.33 \pm 0.45) \times 10^{-11}$ \\
\hline 1438 & 2228 & $(2.31 \pm 0.53) \times 10^{-11}$ \\
\hline 2228 & 3450 & $(2.08 \pm 0.81) \times 10^{-11}$ \\
\hline \multicolumn{3}{|c|}{$2006 / 04 / 30$} \\
\hline 250 & 572 & $(4.87 \pm 1.09) \times 10^{-11}$ \\
\hline 572 & 1310 & $(1.01 \pm 0.21) \times 10^{-10}$ \\
\hline 1310 & 3000 & $(1.01 \pm 0.33) \times 10^{-10}$ \\
\hline \multicolumn{3}{|c|}{$2006 / 06 / 14$} \\
\hline 450 & 669 & $(2.76 \pm 0.74) \times 10^{-11}$ \\
\hline 669 & 995 & $(1.61 \pm 0.67) \times 10^{-11}$ \\
\hline 995 & 1480 & $(2.54 \pm 0.79) \times 10^{-11}$ \\
\hline 1480 & 2200 & $(1.80 \pm 0.85) \times 10^{-11}$ \\
\hline
\end{tabular}

Notes. The two energy bounds specify the range in which the corresponding flux was measured. 


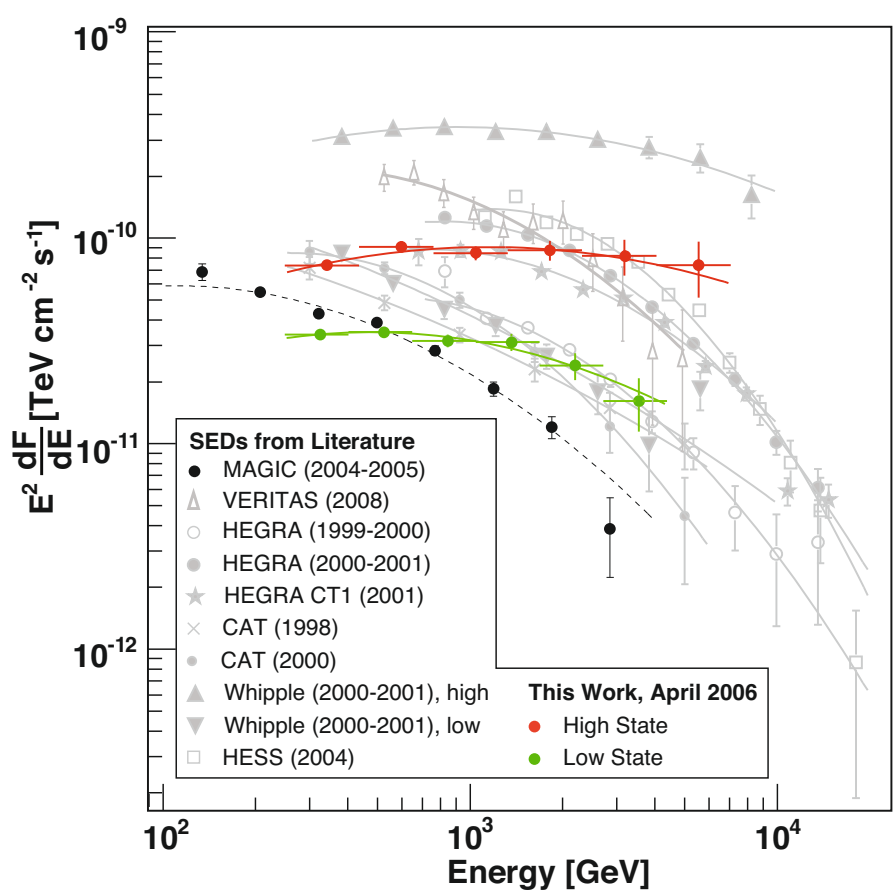

Fig. 7. EBL de-absorbed historical spectra of Mkn 421 (see Albert et al. 2007a, for references) along with selected spectra from the April 2006 campaign and the flare spectrum of Donnarumma et al. (2009). The solid line is the result of a fit using Eq. (4). Note that the historical data were deabsorbed using the model of Primack et al. (2005), our data and those from Donnarumma et al. (2009) with the model of Kneiske \& Dole (2008).

In summary, we followed the evolution of a sequence of mild flares of the blazar Mkn 421 during one week from April 22 to 30,2006 , peaking at $F(E>250 \mathrm{GeV})=(3.21 \pm 0.15) \times$ $10^{-10} \mathrm{~cm}^{-2} \mathrm{~s}^{-1}(\approx 2.0$ Crab units). The nocturnal observations lasted at least for about one hour and allowed for the reconstruction of night-by-night spectra. During three observation nights high fluxes were recorded, in which, however, no variability could be measured. In two of these nights, rather hard spectral indices were found, but this was also the case for the night with the lowest flux. During the night of April 29, 2006, with a not particularly high flux of $F(E>250 \mathrm{GeV})=(1.04 \pm 0.06) \times$ $10^{-10} \mathrm{~cm}^{-2} \mathrm{~s}^{-1}(\approx 0.65$ Crab units $)$, clear intra-night variability with a flux-doubling time of $36 \pm 10_{\text {stat }}$ min was observed.

According to a likelihood ratio test, the spectra of some data sets were better described by curved power laws than simple power laws, enabling us to calculate peak and cutoff energies in the VHE regime. The derived peak values are consistent with an evolution of the peak energy with the flux, as suggested by historical data. Indications of an intrinsic cutoff in the spectra of Mkn 421, as found in former observations, are confirmed by our results.

During the INTEGRAL-triggered MWL campaign in June 2006 we observed Mkn 421 in one night at high zenith angles. Our measurements complement the three-night observations conducted by the Whipple 10-m telescope four days later. Taking the MAGIC and Whipple results together, a variability of Mkn 421 also during the INTEGRAL observations is evident. The energy coverage of the Whipple telescope spectrum $(\Delta E \approx 600 \mathrm{GeV})$ was not sufficient to assess any spectral evolution by comparing it to the MAGIC spectrum $(\Delta E \approx 2 \mathrm{TeV})$.
The determined fluxes and spectra will be further used for studies of the SED taking into account data taken at other photon energies in detailed MWL analyses (publications in preparation).

Acknowledgements. We thank the Instituto de Astrofísica de Canarias for the excellent working conditions at the Observatorio del Roque de los Muchachos in La Palma. The support of the German BMBF and MPG, the Italian INFN and Spanish MICINN is gratefully acknowledged. This work was also supported by ETH Research Grant TH 34/043, by the Polish MNiSzW Grant N N203 390834 , and by the YIP of the Helmholtz Gemeinschaft.

\section{References}

Acciari, V. A., Aliu, E., Aune, T., et al. (VERITAS and MAGIC Collabs.) 2009, ApJ, 703, 169

Aharonian, F., Akhperjanian, A., Beilicke, M., et al. (HEGRA Collab.) 2002, A\&A, 393, 89

Aharonian, F., Akhperjanian, A., Beilicke, M., et al. (HEGRA Collab.) 2004, ApJ, 614, 897

Aharonian, F., Akhperjanian, A. G., Aye, K.-M., et al. (HESS Collab.) 2005, A\&A, 437, 95

Albert, J., Aliu, E., Anderhub, H., et al. (MAGIC Collab.) 2006, ApJ, 638, L101 Albert, J., Aliu, E., Anderhub, H., et al. (MAGIC Collab.) 2007a, ApJ, 663, 125 Albert, J., Aliu, E., Anderhub, H., et al. (MAGIC Collab.) 2007b, Nucl. Instrum. Meth., A583, 494

Albert, J., Aliu, E., Anderhub, H., et al. (MAGIC Collab.) 2008a, ApJ, 674, 1037 Albert, J., Aliu, E., Anderhub, H., et al. (MAGIC Collab.) 2008b, Nucl. Instrum. Meth., A588, 424

Albert, J., Aliu, E., Anderhub, H., et al. (MAGIC Collab.) 2008c, Nucl. Instrum. Meth., A594, 407

Aliu, E., Anderhub, H., Antonelli, L. A., et al. (MAGIC Collab.) 2009, Astropart. Phys., 30, 293

Amati, L., Frontera, F., Tavani, M., et al. 2002, A\&A, 390, 81

Baixeras, C., Bastieri, D., Bigongiari, C., et al. (MAGIC Collab.) 2004, Nucl. Instrum. Meth., A518, 188

Błażejowski, H., Blaylock, G., Bond, I. H., et al. 2005, ApJ, 630, 130

Bretz, T., \& Wagner, R. 2003, in Proc. 28th International Cosmic Ray Conference, Tsukuba, Japan, 5, 2947

Bretz, T., \& Dorner, D. (MAGIC Collab.) 2008, AIP Conf. Proc., 1085, 664

Cortina, J., Armada, A., Biland, A., et al. (MAGIC Collab.) 2005, in Proc. 29th International Cosmic Ray Conference, Pune, India, 5, 359

Cortina, J., Goebel, F., \& Schweizer, T. (MAGIC Collab.) 2009, in Proc. 31st International Cosmic Ray Conference, Łódź, Poland, [arXiv: 0907.1211]

Costamante, L., \& Ghisellini, G. 2002, A\&A, 384, 56

Daum, A., Hermann, G., Hess, M., et al. (HEGRA Collab.) 1997, Astropart. Phys., 8, 1

Donnarumma, I., Vittorini, V., Vercellone, S., et al. (AGILE Collab., GASPWEBT Collab., MAGIC Collab., VERITAS Collab.) 2009, ApJ, 691, L13

Fegan, D. J. 1997, J. Phys. G, 23, 1013

Fegan, D. J. (VERITAS Collab.) 2008, in Proc. 30th International Cosmic Ray Conference, Merida, Mexico, 3, 901

Fomin, V. P., Stepanian, A. A., Lamb, R. C., et al. 1994, Astropart. Phys., 2, 137 Fossati, G., Buckley, J. H., Bond, I. H., et al. 2008, ApJ, 677, 906

Gaidos, J. A., Akerlof, C. W., Biller, S., et al. 1996, Nature, 383, 319

Goebel, F., Backes, M., Bretz, T., et al. (MAGIC Collab.) 2008a, in Proc. 30th International Cosmic Ray Conference, Merida, Mexico, 3, 1025

Goebel F., Bartko, H., Carmona, E., et al. (MAGIC Collab.) 2008b, in Proc. 30th International Cosmic Ray Conference, Merida, Mexico, 3, 1481

Gould, R. J., \& Schréder, G. P. 1966, Phys. Rev. Lett., 16, 252

Hauser, M. G., \& Dwek, E. 2001, ARA\&A, 39, 249

Hillas, A. M. 1985, in Proc. 19th International Cosmic Ray Conference, La Jolla, 3,445

Horan, D., Acciari, V. A., Bradbury, S. M., et al. (Whipple Collab.) 2009, ApJ, 695,596

Hsu, C.-C., et al. (MAGIC Collab.) 2009, in Proc. 31st International Cosmic Ray Conference, Łódź, Poland, [arXiv: 0907.0893]

Katarzyński, K., Ghisellini, G., Mastichiadis, A., Tavecchio, F., \& Maraschi, L. 2006, A\&A, 453, 47

Knapp, J., \& Heck, D. 2004, EAS Simulation with CORSIKA: A Users Manual Kneiske, T. M., \& Dole, H. 2008, AIP Conf. Proc., 1085, 620

Krennrich, F., Bond, I. H., Bradbury, S. M., et al. 2002, ApJ, 575, L9

Krawczynski, H., Sambruna, R., Kohnle, A., et al. 2001, ApJ, 559, 187

Jansen, B., Lumb, D., Altieri, B., et al. 2001, A\&A, 365, 1

Lessard, R. W., Buckley, J. H., Connaughton, V., \& Le Bohec, S. 2001, Astropart. Phys., 15, 1

Li, T.-P., \& Ma, Y.-Q. 1983, ApJ, 272, 317

Lichti, G. G., Bottacini, E., Ajello, M., et al. 2008, A\&A, 486, 721 
Mannheim, K, Westerhoff, S., Meyer, H. \& Fink, H.-H. 1996, A\&A, 315, 77 Majumdar, P., Moralejo, A., Bigongiari, C., Blanch, O., \& Sobczynska, D. 2005, in Proc. 29th International Cosmic Ray Conference, Pune, India, 5, 203 Marscher, A. P., \& Gear, W. K. 1985, ApJ, 298, 11

Maraschi, L., Ghisellini, G., \& Celotti, A. 1992, ApJ, 397, L5

Massaro, E., Perri, M., Giommi, P., \& Nesci, R. 2004, A\&A, 413, 489

Mazin, D., \& Goebel, F. 2007, ApJ, 655, L13

Mazin, D., Goebel, F., Horns, D., et al. 2005, in Proc. 29th International Cosmic Ray Conference, Pune, India, 4, 331

Meucci, M., Paoletti, R., Cecchi, R., et al. 2007, IEEE Trans. Nucl. Sci., 54, 404 Mitsuda, K., Bautz, M., Inoue, H., et al. 2007, PASJ, 59, S1

Mücke, A., Protheroe, R. J., Engel, R., Rachen, J. P., \& Stanev, T. 2003, Astropart. Phys., 18, 593

Nikishov, A. I. 1962, Sov. Phys. JETP, 14, 393

Petry, D., Bradbury, S. M., Konopelko, A., et al. 1996, A\&A, 311, L13

Primack, J. R., Bullock, J. S., \& Somerville, R. S. 2005, AIP Conf. Proc., 745, 23

Punch, M., Akerlof, C. W., Cawley, M. F., et al. 1992, Nature, 358, 477

Riegel, B., Bretz, T., Dorner, D., Berger, K., \& Höhne, D. (MAGIC Collab.) 2005, in Proc. 29th International Cosmic Ray Conference, Pune, India, 5, 215

Sakamoto, T., Barbier, L., Barthelmy, S. D., et al. 2006, ApJ, 636, L73

Schweizer, T., Wagner, R. M., \& Lorenz, E. 2008, AIP Conf. Proc., 1085, 455

Steele, D., Carini, M. T., Charlot, P., et al. (VERITAS Collab.) 2008, in Proc. 30th International Cosmic Ray Conference, Merida, Mexico, 3, 989

Tluczykont M., Shayduk, M., Kalekin, O., \& Bernardini, E. 2007, J. Phys. Conf. Ser., 60, 318

Tonello, N. 2006, Ph.D. Thesis, Technische Universität München, MPP-2006-21

Tramacere, A. 2009, PoS(extremesky2009), 96, 39 [arXiv: 1003.5001]

Wagner, R. M. 2008a, MNRAS, 385, 119

Wagner, R. M. 2008b, PoS(BLAZARS2008), 63, 13 [arXiv:0808.2483]

Yadav, K. K., Chandra, P., Tickoo, A. K., et al. (TACTIC Collab.) 2007, Astropart. Phys., 27, 447

1 IFAE, Edifici Cn., Campus UAB, 08193 Bellaterra, Spain

2 ETH Zurich, 8093, Switzerland

3 INAF National Institute for Astrophysics, 00136 Rome, Italy
4 Universidad Complutense, 28040 Madrid, Spain e-mail: oya@gae.ucm.es

5 Technische Universität Dortmund, 44221 Dortmund, Germany

6 Universitat Autònoma de Barcelona, 08193 Bellaterra, Spain

7 Università di Padova and INFN, 35131 Padova, Italy

8 Inst. de Astrofísica de Canarias, 38200 La Laguna, Tenerife, Spain

9 University of Łódź, 90236 Łódź, Poland

10 Tuorla Observatory, University of Turku, 21500 Piikkiö, Finland

11 Deutsches Elektronen-Synchrotron (DESY), 15738 Zeuthen, Germany

12 Max-Planck-Institut für Physik, 80805 München, Germany e-mail: robert.wagner@mpp.mpg.de

13 Università di Siena, and INFN Pisa, 53100 Siena, Italy

14 Universitat de Barcelona (ICC/IEEC), 08028 Barcelona, Spain

15 Universität Würzburg, 97074 Würzburg, Germany e-mail: snruegam@astro.uni-wuerzburg.de

16 Depto. de Astrofisica, Universidad, 38206 La Laguna, Tenerife, Spain

17 Università di Udine, and INFN Trieste, 33100 Udine, Italy

18 Institut de Ciències de l'Espai (IEEC-CSIC), 08193 Bellaterra, Spain

19 Inst. de Astrofísica de Andalucía (CSIC), 18080 Granada, Spain

20 Croatian MAGIC Consortium, Institute R. Boskovic, University of Rijeka and University of Split, 10000 Zagreb, Croatia

21 University of California, Davis, 95616-8677, USA

22 Inst. for Nucl. Research and Nucl. Energy, 1784 Sofia, Bulgaria

23 INAF/Osservatorio Astronomico and INFN, 34143 Trieste, Italy

24 ICREA, 08010 Barcelona, Spain

25 Università di Pisa, and INFN Pisa, 56126 Pisa, Italy

26 Supported by INFN Padova

27 Now at: Centro de Investigaciones Energéticas, Medioambientales y Tecnológicas (CIEMAT), Madrid, Spain

28 Now at: Max-Planck-Institut für Kernphysik, 69029 Heidelberg, Germany 